\title{
Self-tuning fuzzy logic control for ultrasound hyperthermia with reference temperature based on objective functions
}

\author{
Yung-Yaw Chen \\ Department of Electrical Engineering, National Taiwan University, Taipei, Taiwan \\ Win-Li Lin ${ }^{\text {a) }}$ \\ Institute of Biomedical Engineering, National Taiwan University, Taipei, Taiwan \\ Hau-Li Liou \\ Department of Electrical Engineering, National Taiwan University, Taipei, Taiwan \\ Jia-Yush Yen \\ Department of Mechanical Engineering, National Taiwan University, Taipei, Taiwan \\ Ming-Jium Shieh \\ Institute of Biomedical Engineering, National Taiwan University, Taipei, Taiwan
}

(Received 16 March 1998; accepted for publication 2 March 1999)

\begin{abstract}
The purpose of this paper is to develop and evaluate a self-tuning fuzzy logic controller for a scanned focused ultrasound hyperthermia system with the reference temperature $\left(T_{r}\right)$ determined from objective functions. This work employs simulation programs to develop the power deposition for the scanned focused ultrasound system and to solve the responses of temperature profiles based on the transient bioheat transfer equation. A fuzzy logic control algorithm is employed to determine the output power level for the heating system and an observer for blood perfusion variation is used to enhance the capability of the controller to adjust the required output power level for the treatment due to the drastic change of the blood perfusion. The reference temperature $\left(T_{r}\right)$ for the controller is based on objective functions to tune its value during the heating process, while a control temperature $\left(T_{c}\right)$ from the thermosensors located in the tumor region is used as the input for the controller. The objective function based on the entire temperature profile is used to evaluate the appropriateness of the heating temperature distribution for a time-variational blood perfusion. Simulation results demonstrate that the tumor region can be rapidly heated to the desired temperature level and maintained at that level despite blood perfusion variation. The resulting temperature profile, the objective function, and the output power level are related to the magnitude of blood perfusion, but are almost independent of the $T_{c}$ location and the initial setting value of $T_{r}$. The fuzzy logic control algorithm with $T_{r}$ determined from objective functions can be used for controlling the entire temperature distribution through a single control temperature, and the combination of control and optimization allows appropriate temperature fields to be created during the entire heating process. The control algorithm does not require the accurate prior knowledge of the locations of the thermosensors and the appropriate setting value for $T_{r}$. (C) 1999 American Association of Physicists in Medicine. [S0094-2405(99)01805-2]
\end{abstract}

Key words: self-tuning fuzzy logic controller, objective function, reference temperature, control temperature, scanned focused ultrasound hyperthermia

\section{INTRODUCTION}

The goal of hyperthermia treatment is to calculate the complete temperature field, to adjust power deposition parameters, and then to optimize the proposed thermal treatment by maximizing the therapeutic effects of the tumor temperature distribution while minimizing normal tissue damage and patient stress. ${ }^{1}$ To achieve this goal, several investigators have proposed and/or applied control systems to hyperthermia treatments. For example, Fenn and Gerald ${ }^{2}$ investigated a computer-controlled adaptive phased-array radio-frequency hyperthermia system for improved therapeutic tumor heating. The steepest decent algorithm is applied to derive the optimal power levels for several channels within the region of the tumor and neighboring normal tissues. The measured data indicate that with an adaptive hyperthermia array it may be possible to maximize the applied electric field at a tumor position and simultaneously minimize the occurrences of hot spots at the target position. Van Baren et al. ${ }^{3}$ developed a feedback control algorithm for control of tumor temperature during phased-array ultrasound hyperthermia treatments. They set different desired temperatures as the reference temperatures for some thermosensors and the temperatures induced by the specified power patterns are mutually decoupled. Based on a simplified state-space model for the bioheat transfer equation and the least-squared error to evaluate the treatment, the tumor heating was controlled such that the actual temperatures were as close as possible to the specified temperatures. Potocki and Tharp ${ }^{4}$ used the information from 
an optimization study for a scanned focused ultrasound system to establish a schedule of desirable scan patterns as a function of the estimated blood perfusion magnitude. They found the thermal performance of the resulting closed-loop system is strongly influenced by the locations of the measured temperatures and the regulated power outputs. Finally, Lin and co-workers ${ }^{5,6}$ employed an optimization scheme and a control algorithm to control a two-dimensional (2D) temperature field for a scanned focused ultrasound system to overcome the blood perfusion variation. When applying the control algorithm to determined the magnitude of output power, they found that the locations and the target temperature of control thermocouples had significant influence on the resulting temperature distribution and the output power level due to the Gaussian shape of the ultrasound power deposition combined with the circular scan path.

The goal of the above control algorithms mainly focused on the control of the thermosensors' temperatures to the given values ${ }^{3,5}$ or on the occurrences of hot spots in the normal tissue while applying the maximum power in the tumor. ${ }^{2}$ However, the thermal performance would be strongly influenced by the locations of the measured temperatures and/or the given values for the control thermosensors. ${ }^{4-6}$ The proposed control algorithm with a reference temperature $\left(T_{r}\right)$ based on objective functions can produce a temperature distribution which is able to optimize the proposed thermal treatment ${ }^{1}$ during the entire heating process, while the heating result is not significantly influenced by the control thermosensor locations or the given values of the reference temperatures.

The blood perfusion distribution is known to be one of the most important factors that affects temperature distributions in hyperthermia. A parametric study has been done ${ }^{7}$ and the results show that appropriate temperature distributions can be obtained for a large range of spatial variations for blood perfusion $\left(1-10 \mathrm{~kg} \mathrm{~m}^{-3} \mathrm{~s}^{-1}\right.$ for the normal tissue and $0-5$ $\mathrm{kg} \mathrm{m}^{-3} \mathrm{~s}^{-1}$ for the tumor tissue) using optimization techniques. However, blood perfusion is also a time-variational unknown during the hyperthermia treatment; it is important to include some form of feedback control to compensate for this factor. Hence, the objective of this work is to develop a control algorithm to obtain the optimal temperature distribution within the heating region with constraint on the output measured temperatures and containing blood perfusion that is initially unknown and/or varies with time. A uniform perfusion for the tumor and normal tissue is assumed and an optimization method ${ }^{7}$ is employed to search for the set of scan parameters which can form a power deposition conformal to the tumor region. A self-tuning fuzzy logic controller with the reference temperature $\left(T_{r}\right)$ based on objective functions is employed to tune the power level to meet the treatment requirements. This fuzzy controller with this type of $T_{r}$ can overcome the blood perfusion variation and drive the heating temperature field to the optimal condition, which is independent of the thermosensor location of the control temperature $\left(T_{c}\right)$ and the initial setting value of $T_{r}$.

\section{METHOD}

\section{A. Ultrasound power development}

Figure 1(a) shows the geometry of an ultrasound heating system with a single scanning transducer. ${ }^{7,8}$ Power is emitted from the transducers arranged below the treatment volume. The four scan parameters (tilt angle, rotation angle, focal depth, and scan radius) for the transducer that can be varied to obtain an appropriate heating configuration are shown. The magnitude of ultrasound output power can also be varied. When the transducer scanning speed is sufficient, the power deposition patterns could be considered symmetrical about the $z$ axis for tissues with homogeneous ultrasound absorption properties. To calculate the absorbed power distribution, first the power deposition pattern for a stationary transducer was calculated and the programs developed by Moros ${ }^{9}$ and $\operatorname{Lin}^{7}$ were used to tilt and rotate the stationary power deposition through given angles. Next, the power field was translated away from the central scan axis a distance equal to the scan circle radius to develop a power deposition pattern for a given scan radius. Finally a convolution integral of the stationary pattern was used to obtain the average power at each node to account for the scanning process. This program also converts the three-dimensional power field,
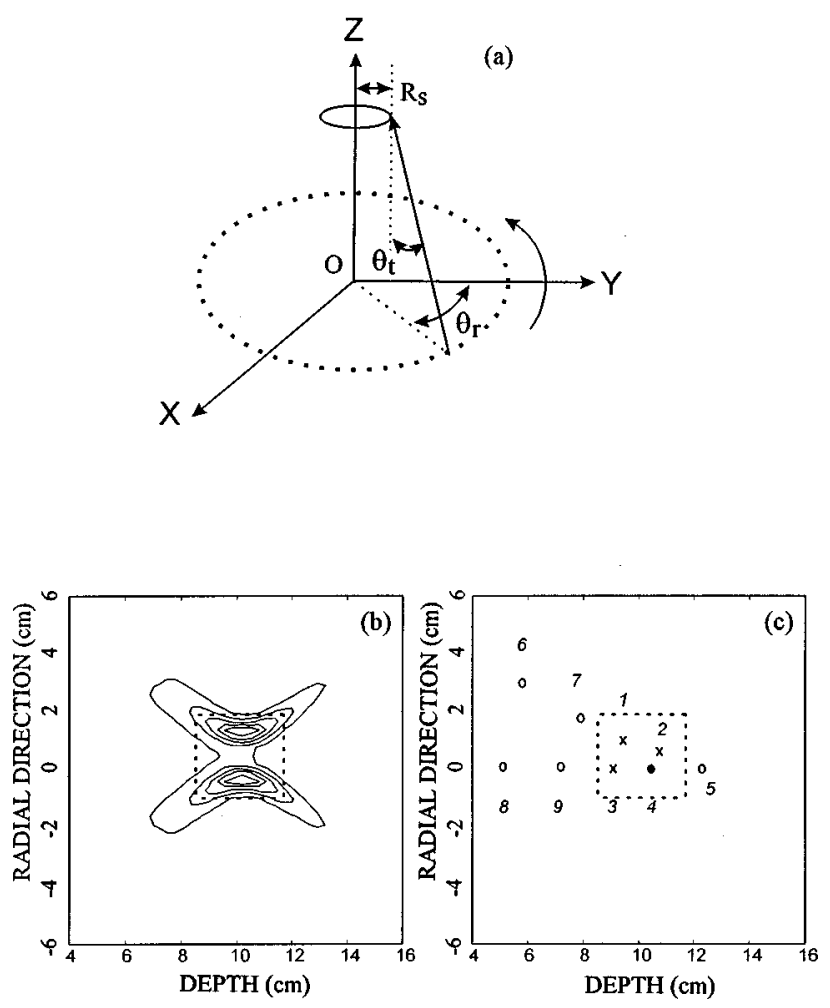

FIG. 1. (a) The geometry of a scanned focused ultrasound heating system; (b) the normalized power deposition for the set of scan parameters [tile angle $\left(\theta_{t}\right) 40^{\circ}$, rotation angle $\left(\theta_{r}\right) 90^{\circ}$, scan radius $\left(R_{s}\right) 1.0 \mathrm{~cm}$, focal depth $10 \mathrm{~cm}$ (the distance between the skin and the focal plane)], and the transducer (radius of curvature $20 \mathrm{~cm}$, diameter $10 \mathrm{~cm}$, and frequency $1.0 \mathrm{MHz}$ ); (c) a $3 \mathrm{~cm} \times 3 \mathrm{~cm}$ cylindrical tumor located in the central region and the thermosensor locations are shown. 


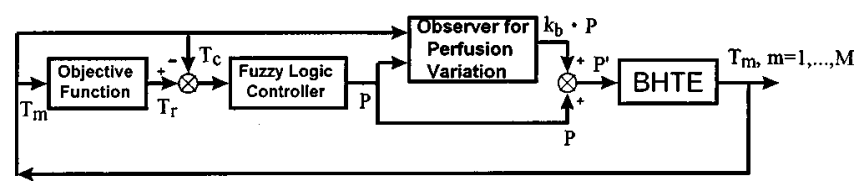

FIG. 2. Conceptual block diagram of the self-tuning fuzzy logic controller with an observer for the blood perfusion variation and the reference temperature $\left(T_{r}\right)$ as defined by Eq. (5).

which is in Cartesian coordinates for the above tilting, rotating, and translating, into a 2D field in cylindrical coordinates. Details of the above procedures are given by Lin. $^{7} \mathrm{An}$ ultrasound transducer with a diameter of $10 \mathrm{~cm}$, radius of curvature of $20 \mathrm{~cm}$, and driving frequency of $1.0 \mathrm{MHz}$ has been chosen for this study. To obtain a simple but realistic approximation of the power deposition, the ultrasound attenuation in tissue wa assumed to be an average of $10 \mathrm{~Np} / \mathrm{m}$ for all simulations, and the power generated from all parts of the transducer was assumed to be attenuated equally at each depth. ${ }^{7,9}$

\section{B. Temperature solver}

To solve for the temperature distribution, Pennes ${ }^{10}$ transient bioheat transfer equation (BHTE) was used;

$$
\rho c \frac{\partial T}{\partial t}=-k \nabla^{2} T+W c_{b}\left(T-T_{\mathrm{ar}}\right)+Q_{p} .
$$

We chose the thermal properties to approximate averages for soft tissues. ${ }^{11-13}$ The thermal conductivity $(k)$ is 0.5 ( $\mathrm{W} \mathrm{m}^{-1}{ }^{\circ} \mathrm{C}^{-1}$ ), the specific heat of blood $\left(c_{b}\right)$ is $3770(\mathrm{~J}$ $\left.\mathrm{kg}^{-1}{ }^{\circ} \mathrm{C}^{-1}\right)$, and the arterial temperature $\left(T_{\mathrm{ar}}\right)$ is $37^{\circ} \mathrm{C}$. The density of absorbed ultrasonic power $\left(Q_{p}\right)$ was obtained according to the above power development. A blood perfusion $(W)$ that keeps a constant value of space was used. The anatomic properties were assumed to be constant throughout the entire field, and metabolism was neglected due to its small contribution to the temperature changes. ${ }^{1,14}$ Thus, all cases had symmetrical, two-dimensional field $(r, z)$. The diameter and the thickness of the cylindrical volume of the simulated tissue were both $20 \mathrm{~cm}$. The boundary condition for the front end was a constant value, which was equal to the temperature of the surface cooling water, and the other surface was a constant temperature $37^{\circ} \mathrm{C}$. The central axis was treated as adiabatic $^{15,16}$ because no energy transfer occurs in the radial direction at the center line due to the symmetry of both the power distribution and the anatomical properties.

\section{Self-tuning fuzzy logic control algorithm}

An overall control scheme is schematically shown in Fig. 2. A fuzzy logic controller with a blood perfusion variation observer is employed to determine the output power level for the heating system, and the control temperature $\left(T_{c}\right)$ for the controller is selected from the thermosensors located within the tumor region. A time-varying reference temperature $\left(T_{r}\right)$, which is based on objective functions associated with constraints on temperature in the tumor and normal tissues, is

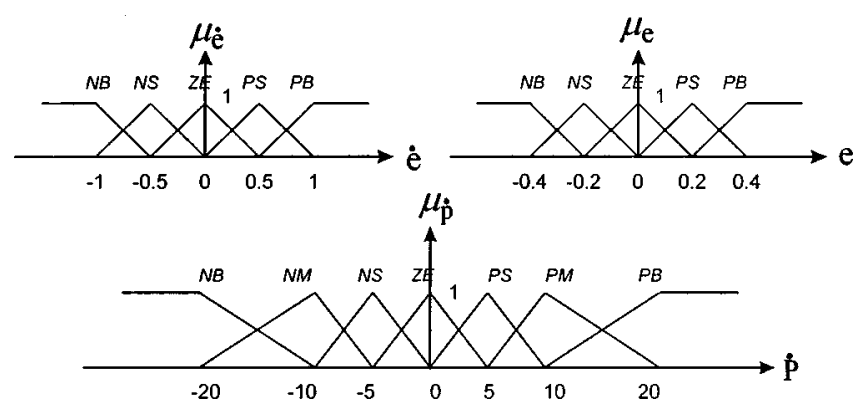

FIG. 3. The input and output membership functions of the fuzzy sets for the self-tuning fuzzy logic controller. The fuzzy set NB means negative big; NM, negative medium; NS, negative small; ZE, zero; PS, positive small; $\mathrm{PM}$, positive medium; and $\mathrm{PB}$, positive big.

used as the target for $T_{c}$. The initial value for $T_{r}$ is set between 43 and $46^{\circ} \mathrm{C}$. The sampling time for all simulations is $30 \mathrm{~s}$. During the first five minutes (ten steps), the bangbang control action and a fixed $T_{r}$ are employed to give a fast rise time by using the maximum permissible power when $T_{c}$ is low.

\section{Design of self-tuning fuzzy logic controller}

In this study, a self-tuning fuzzy logic controller that is sufficiently robust over the ultrasound heating process is used. ${ }^{17,18}$ The temperature different $(e)$ between $T_{r}$ and $T_{c}$, as well as the derivative $(\dot{e})$ of the temperature difference, are regarded as the input variables for the fuzzy logic controller, while the derivative $(\dot{p})$ of the output power $(p)$ for the controller is taken as the output variable. The fuzzy logic rule taken is

$$
R_{i} \text { : } \quad \text { if } \dot{e} \text { is } A_{i} \text { and } e \text { is } B_{i} \text { then } \dot{p} \text { is } C_{i} .
$$

The membership functions of fuzzy sets for the inputs and output are shown in Fig. 3, and Table I is used as the control rule base. This is a PI-like (proportional-integral) fuzzy logic controller.

To overcome the problem caused by variation of blood perfusion more effectively during the treatment process, ${ }^{19,20}$ a self-tuning mechanism is employed to observe the change of blood perfusion and modify the output power level for the fuzzy logic controller. The derivatives $\left(\dot{p}, \dot{T}_{c}\right)$ of the output power and the control temperature together with the difference $(e)$ between $T_{r}$ and $T_{c}$ are regarded as the input variables for the self-tuning mechanism. A scale $\left(k_{b}\right)$ is taken as the output to more precisely adjust the output power level for

TABLE I. The rule base for the fuzzy logic controller.

\begin{tabular}{cccccc}
\hline \hline$e \backslash \frac{\dot{e}}{\dot{P}}$ & NB & NS & ZE & PS & PB \\
\hline NB & NB & NB & NM & NS & ZE \\
NS & NB & NM & NS & ZE & PS \\
ZE & NM & NS & ZE & PS & PM \\
PS & NS & ZE & PS & PM & PB \\
PB & ZE & PS & PM & PB & PB \\
\hline \hline
\end{tabular}




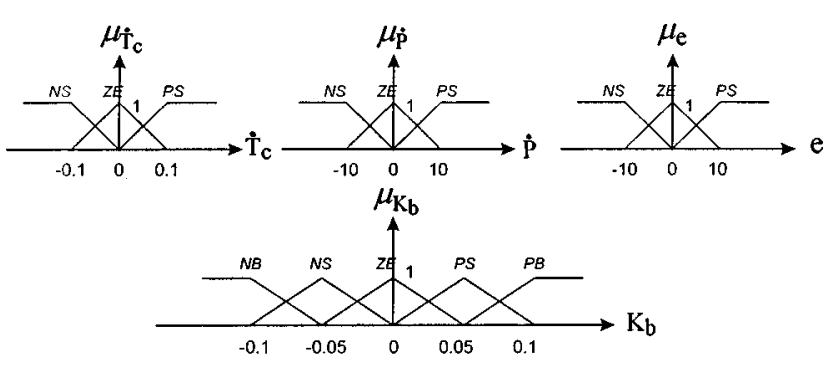

FIG. 4. The input and output membership functions of the fuzzy sets for the self-tuning mechanism used as the blood perfusion variation observer.

the controller to meet the requirement of the dramatic blood perfusion variation. The self-tuning mechanism rule is defined as

$$
\begin{aligned}
& R_{j}: \quad \text { if } \dot{T}_{c}(n-1) \text { is } A_{j} \text { and } \dot{p}(n-1) \text { is } B_{j} \\
& \text { and } e(n-1) \text { is } C_{j} \text { then } k_{b}(n) \text { is } D_{j},
\end{aligned}
$$

where

$$
\begin{aligned}
& \dot{T}_{c}(n-1)=T_{c}(n-1)-T_{c}(n-2), \quad \text { and } \\
& \dot{p}(n-1)=p(n-1)-p(n-2) .
\end{aligned}
$$

The membership functions for the inputs and output are shown in Fig. 4, and Table II is the self-tuning mechanism rule base.

\section{Objective functions and reference temperature}

In order to achieve an optimal temperature field for the treatment region during the treatment process, a treatment goal must be defined. Subsequently, a suitable control algorithm is employed to appropriately vary the output power level when the ultrasound heating system is arranged according to the optimal scan parameters obtained from an optimization algorithm. Conventionally, the treatment goal is to set an appropriate reference temperature for the control thermosensors, ${ }^{3,5}$ and then use a control algorithm to tune the output power level for the heating system based on the difference between the reference and the control temperatures. The treatment goal given here is to heat the treatment region and maintain the temperature as close as possible to an ideal temperature distribution by minimizing an appropriate objective function during the treatment. ${ }^{7}$ The ideal temperature

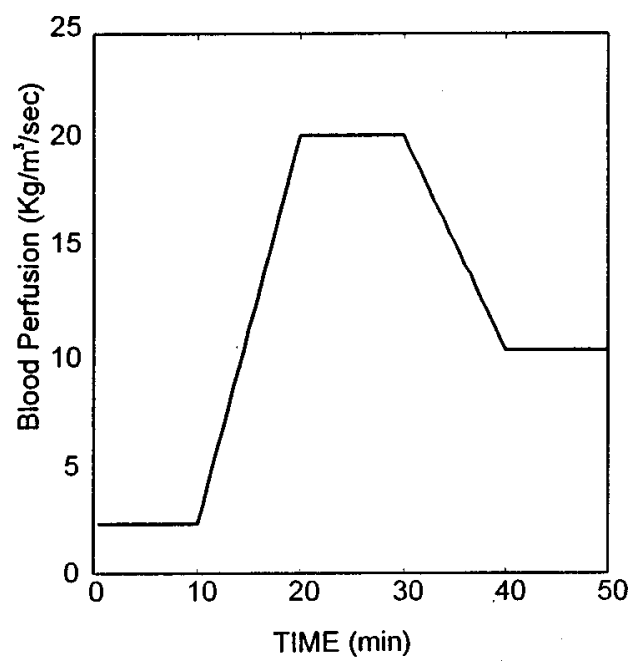

FIG. 5. The temporal variation of blood perfusion during the heating process.

distribution defined here for the treatment region is a temperature profile between 43 and $46{ }^{\circ} \mathrm{C}$ for the tumor, and a maximum temperature for the normal tissue lower than $40{ }^{\circ} \mathrm{C}$. Thus, the control algorithm chosen here is one which attempts to achieve this goal during the entire treatment process by minimizing the following least-squared objective function with a time-varying reference temperature:

$$
J(n)=\left[J_{1}(n)+J_{2}(n)\right] / M,
$$

where

$$
J_{1}(n)=\Sigma\left[T_{i}(n)-46\right]^{2}+\Sigma\left[T_{j}(n)-40\right]^{2},
$$

with $T_{i} \in$ tumor and $>46^{\circ} \mathrm{C}$,

$T_{j} \in$ normal tissue and $>40{ }^{\circ} \mathrm{C}$,

$$
J_{2}(n)=\Sigma\left[T_{k}(n)-43\right]^{2},
$$

with $T_{k} \in$ tumor and $<43{ }^{\circ} \mathrm{C}$,

where $J(n)$ is the objective function based on the measured temperatures of the tumor and normal tissues; $J_{1}(n)$ and $J_{2}(n)$ represent the subobjective functions for the tumor/ normal tissue temperature higher than and the tumor temperature lower than the requirements, respectively; $M$ repre-

TABLE II. The rule base for the self-tuning mechanism used as the variation observer for blood perfusion.

\begin{tabular}{llllllllllll}
\hline \hline$\dot{T}_{c}$ & $\dot{P}$ & $e$ & $K_{b}$ & $\dot{T}_{c}$ & $\dot{P}$ & $e$ & $K_{b}$ & $\dot{T}_{c}$ & $\dot{P}$ & $e$ & $K_{b}$ \\
\hline NS & NS & NS & PS & ZE & NS & NS & PS & PS & NS & NS & ZE \\
NS & NS & ZE & ZE & ZE & NS & ZE & ZE & PS & NS & ZE & NS \\
NS & NS & PS & NS & ZE & NS & PS & NS & PS & NS & PS & PB \\
NS & ZE & NS & PS & ZE & ZE & NS & PS & PS & ZE & NS & ZE \\
NS & ZE & ZE & ZE & ZE & ZE & ZE & ZE & PS & ZE & ZE & ZE \\
NS & ZE & PS & ZE & ZE & ZE & PS & ZE & PS & ZE & PS & NS \\
NS & PS & NS & PB & ZE & PS & NS & PS & PS & PS & NS & PS \\
NS & PS & ZE & PS & ZE & PS & ZE & ZE & PS & PS & ZE & ZE \\
NS & PS & PS & ZE & ZE & PS & PS & ZE & PS & PS & PS & NS \\
\hline \hline
\end{tabular}



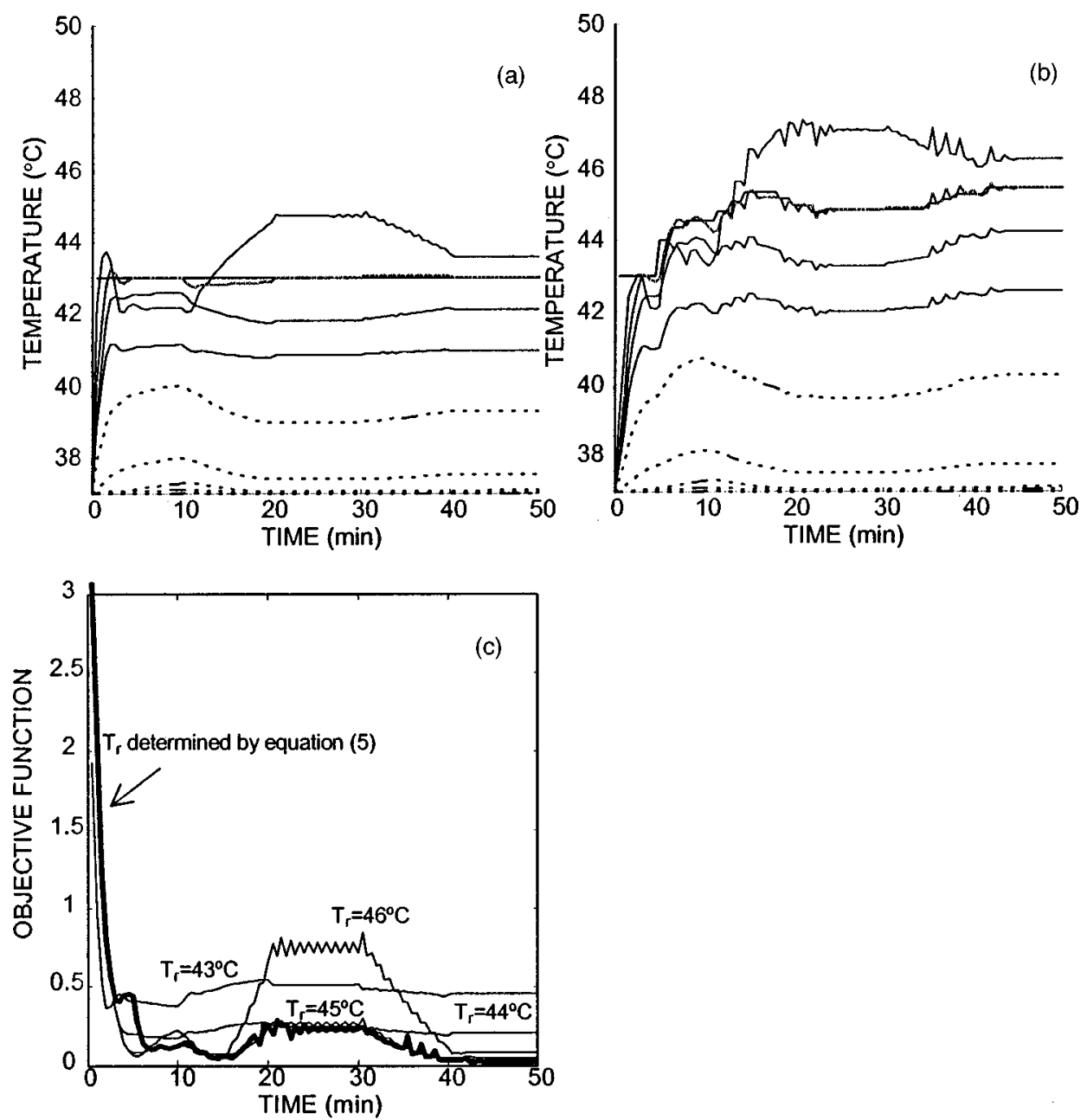

Fig. 6. Effect of the reference temperature $\left(T_{r}\right)$ on the temperature profiles and the objective function. (a) is the temperature profile for a fixed value of $T_{r}=43{ }^{\circ} \mathrm{C}$. The solid and the dashed curves are for the thermosensors located in the tumor and normal tissue regions, respectively; the horizontal line is for the fixed $T_{r}$; and the solid curve with open symbols is for $T_{c}$. (b) is the temperature profile for $T_{r}$ as defined by Eq. (5); the curves for $T_{c}$ and $T_{r}$ are close together during the heating process. (c) is the response of the objective functions for $T_{r}$ as defined by Eq. (5) or kept constant at $43,44,45$, and $46{ }^{\circ} \mathrm{C}$, respectively; the bold curve is for $T_{r}$ as defined by Eq. (5).

sents the total number of thermosensors, $n$ denotes the heating time series, and $i, j, k$, are the indices for the thermosensor locations.

The objective function $J(n)$ is based on the two subobjective functions $J_{1}(n)$ and $J_{2}(n)$, which are associated with constraints on temperature in the tumor and normal tissues (hot/cold spots). To achieve a treatment temperature always close to the ideal temperature distribution during the heating process, a time-varying reference temperature formed by $J_{1}(n)$ and $J_{2}(n)$ with a proper weighting is used for the controller to supply a temporally appropriate power level to overcome the variation of blood perfusion, which is unknown in treatments,

$$
T_{r}(n+1)=T_{r}(n)+k_{p}\left\{\left[J_{2}(n)\right]^{1 / 2}-k_{0}\left[J_{1}(n)\right]^{1 / 2}\right\},
$$

where $T_{r}(n)$ is the reference temperature for the control thermosensor; and $k_{p}, k_{0}$ denote the gain and the weighting of $J_{1}(n)$ and $J_{2}(n)$, respectively.

The gain $\left(k_{p}\right)$ determines the convergence speed, while the weighting $\left(k_{0}\right)$ is related to the final value of the objec- tive function when a controller with this $T_{r}$ is employed. In the following study, $k_{p}$ and $k_{0}$ are taken as 0.5 and 1.0, respectively, which can give a fast convergence speed and a low value of the objective function close to the minimum (using optimization techniques ${ }^{7}$ ).

\section{RESULTS AND DISCUSSION}

A cylindrical tumor with $3 \mathrm{~cm}$ for both diameter and thickness is located at a $10 \mathrm{~cm}$ depth, and a uniform blood perfusion was assumed for both tumor and normal tissue. The scan parameters used are $40^{\circ}$ for tilt angle, $90^{\circ}$ for rotation angle, $1.0 \mathrm{~cm}$ for scan radius, and $10 \mathrm{~cm}$ for the focal depth. Two thermoprobes (with nine measurement locations totally) are arranged: one is along the axial axis for the scanning transducer and the other is along the central axis of the heating volume. Figures 1(b) and 1(c) depict the control volume, the tumor region, the thermosensor locations, and the two-dimensional ultrasound power deposition formed by this set of scan parameters. The blood perfusion is assumed to be 

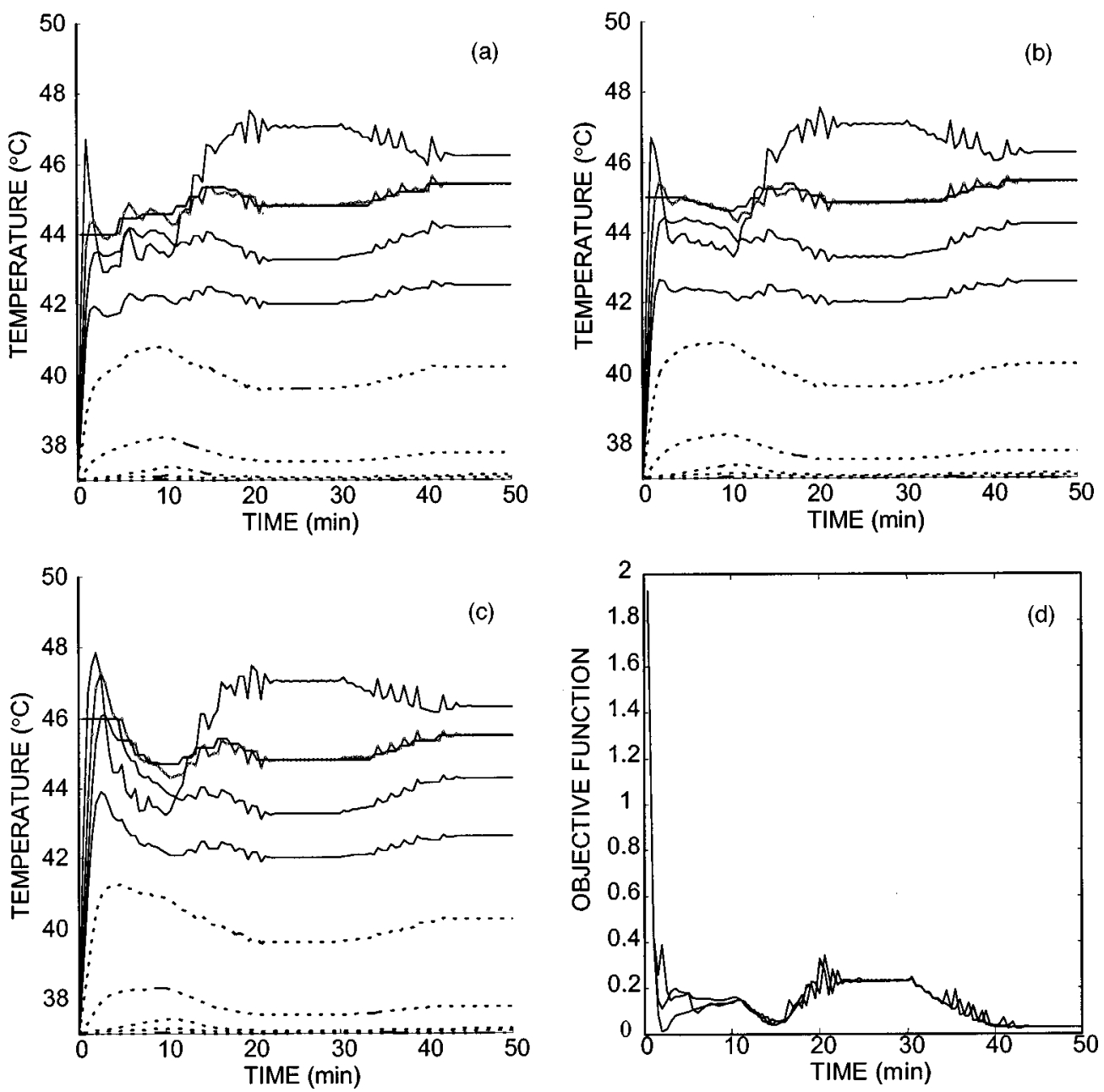

FIG. 7. Effect of the initial setting value of $T_{r}$ on the temperature profiles and the objective functions. (a)-(c) are the response of $T_{r}$ and the temperature profiles for $T_{r}$ initially set at 44,45 , and $46^{\circ} \mathrm{C}$, respectively; the solid and the dashed curves are for the thermosensors within the tumor and the normal tissue regions, respectively; the solid curve with open symbols is for $T_{c}$; and the solid curve close to $T_{c}$ is for $T_{r}$. (d) is the response of the objective functions for $T_{r}$ initially set at 44,45 , and $46^{\circ} \mathrm{C}$, respectively.

uniform within the treatment domain for tumor and normal tissue but varied temporally as shown in Fig. 5. ${ }^{19}$

The responses of the temperature profiles and the objective function are used to evaluate the performance of the self-tuning fuzzy controller with regards to the location of the control temperature $\left(T_{c}\right)$, the setting value of the reference temperature $\left(T_{r}\right)$, and the variation of blood perfusion. Figure 6(a) is the computer simulation result for the response of the temperature profiles when a fixed value of $T_{r}$ at $43{ }^{\circ} \mathrm{C}$ is used for the controller. Figure 6(a) indicates that with a fixed $T_{r}, T_{c}$ can reach $T_{r}$ within a short period of heating time and be maintained within a reasonable range even though the perfusion varies dramatically during the heating process. However, the temperature profile shows that the overall temperature field is influenced by the setting value of $T_{r}$ and a higher $T_{r}$ results in a higher entire temperature profile. This relationship between the temperature profile and the fixed value of $T_{r}$ reveals that the conventional controllers with a fixed $T_{r}$ result in temperature distributions which depend on the value of $T_{r}$ and cannot automatically meet the requirements for the hyperthermia treatment. To reduce the influences of $T_{r}$ and blood perfusion on the overall temperature field and to achieve a better temperature profile for the entire heating process, a modified, time-varying $T_{r}$ as defined by Eq. (5) is employed. Figure 6(b) shows the responses of the control temperature $\left(T_{c}\right)$, the reference temperature $\left(T_{r}\right)$, and the entire temperature profile when $T_{r}$ as defined by Eq. (5) is used for the self-tuning fuzzy logic controller. Figure 6(b) also illustrates that $T_{c}$ follows $T_{r}$ closely, and the measured tumor temperatures move to and then maintain in the range of $43-46{ }^{\circ} \mathrm{C}$ during the heating process. Figure 6(c), which shows the response of the objective functions for this $T_{r}$ as well as fixed $T_{r}$ equal to 43,44 , 45 , and $46{ }^{\circ} \mathrm{C}$, respectively, indicates that the objective function is varied with $T_{r}$ and heating time, and that the response for this $T_{r}$ is always in the lowest level except in the initial stage where the objective function is mainly influenced by the initial setting value of $T_{r}$. After the value of $T_{r}$ is adjusted, the objective function falls to the lowest level. The simulation results give evidence that the self-tuning fuzzy logic controller with $T_{r}$ as defined by Eq. (5) can drive the overall temperature profile to the optimal condition during 

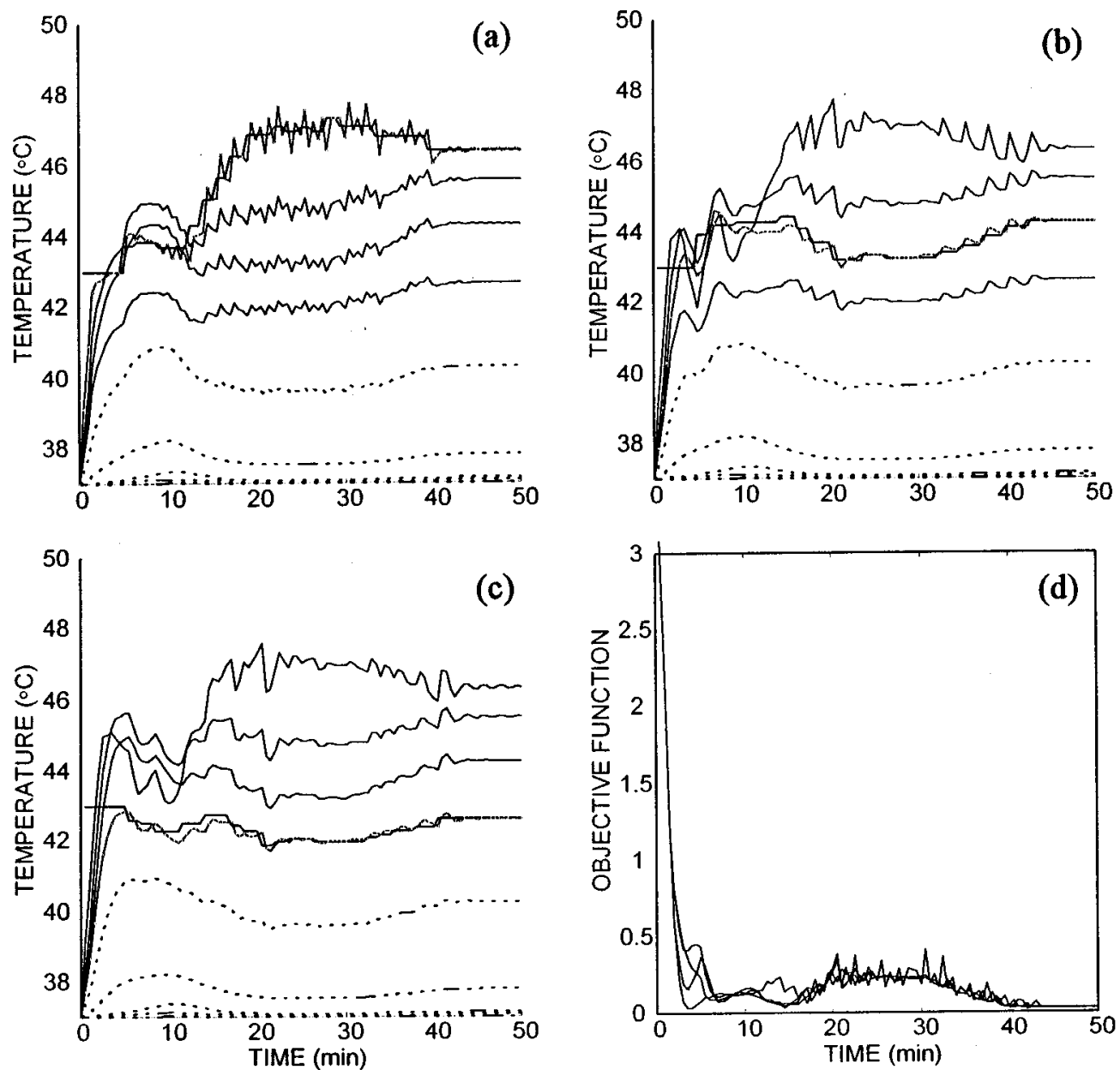

FIG. 8. Effect of the $T_{c}$ location on the reference temperature $\left(T_{r}\right)$, the temperature profiles, and the objective function. (a)-(c) are the response of $T_{r}$ and the temperature profiles for $T_{c}$ located at thermosensors 1,2, and 3, respectively; the solid and the dashed curves are for the thermosensors within the tumor and the normal tissue regions, respectively; the solid curve with open symbols is for $T_{c}$, and the solid curve close to $T_{c}$ is for $T_{r}$. (d) is the response of the objective functions for $T_{c}$ set at thermosensors $1,2,3$, and 4 , respectively.

the entire treatment process instead of only maintaining between $T_{c}$ and $T_{r}$.

The influence of the initial setting value for $T_{r}$ on the entire temperature profile during the heating process is also studied. Figures $7(\mathrm{a})-7(\mathrm{c})$ are the simulation results for the responses of temperature profiles when an initial value for $T_{r}$ at 44,45 , or $46^{\circ} \mathrm{C}$ is used for the controller, respectively. Figure $7(\mathrm{~d})$ shows the objective functions for the initial value of $T_{r}$ set at 44,45 , and $46{ }^{\circ} \mathrm{C}$. These figures demonstrate that the influence of the initial value of $T_{r}$ on the temperature profile only appears in the early part of the heating process. These simulation results indicate that the system performance is not affected by initial setting of $T_{r}$.

To examine the influence of the $T_{c}$ location on $T_{r}$ and the entire temperature profile, the thermosensors 2,3 , and 4 are individually taken as $T_{c}$ with $T_{r}$ set to $43{ }^{\circ} \mathrm{C}$ initially. Figures 8(a)-8(c), which show the response of the temperature profiles and the temporal variations of $T_{c}$ and $T_{r}$ while $T_{c}$ is set to the thermosensors 2,3 , and 4 , respectively, reveal that the entire temperature profiles for all three cases are very close. In addition, $T_{c}$ is able to track $T_{r}$ closely in all cases. Figure $8(d)$, which shows the response of objective functions for thermosensors $1,2,3$, and 4 taken as $T_{c}$, shows that the objective functions for all four cases coincide with one another and remain at the same level except for some minor disturbances over the entire heating process. Both the temperature profile and the objective function obviously demonstrate that the treatment heating result is almost independent of the $T_{c}$ location when $T_{r}$ as defined by Eq. (5) is employed for the self-tuning fuzzy logic controller.

To examine the effects of the blood perfusion and the $T_{c}$ location on the objective function and the output power level, the scan parameters (tilt angle, rotation angle, scan radius, and focal depth) are maintained while the output power level is the only parameter to be tuned to achieve an appropriate temperature distribution. The blood perfusion is maintained constant for the entire volume at a value of 2, 5 or $10 \mathrm{~kg} \mathrm{~m}^{-3} \mathrm{~s}^{-1}$, and the measured temperature for thermosensor $1,2,3$, or 4 is taken as $T_{c}$ for the self-tuning fuzzy logic controller. Figures 9(a)-9(d) present the simulation results showing the responses of the objective functions. The objective functions quickly come to the lowest level region and then are maintained at specific values which are related to the magnitude of the blood perfusion but independent of 

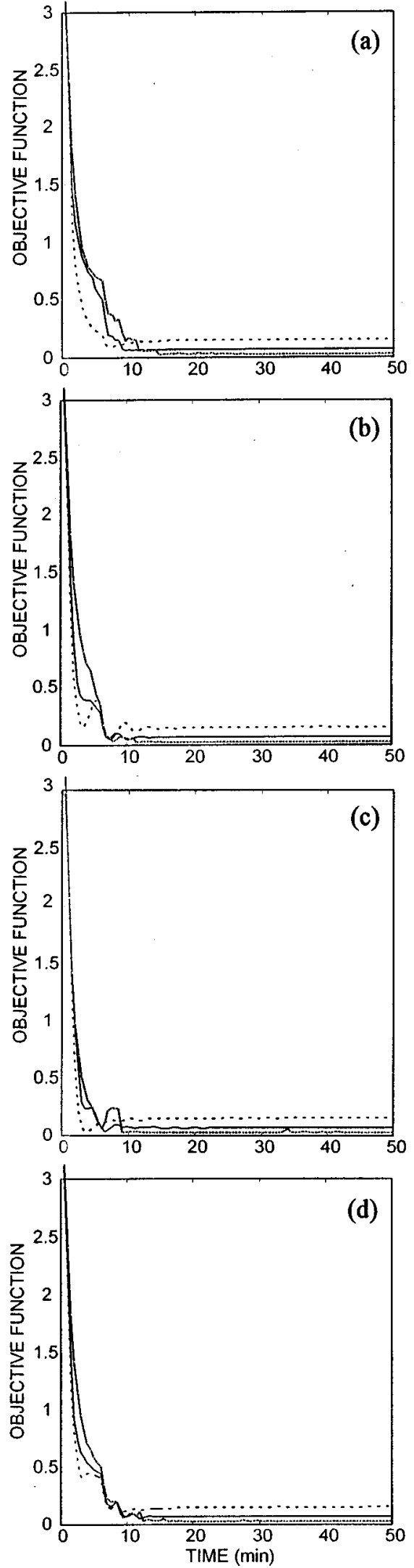

the $T_{c}$ location. A higher perfusion produces a larger value for the objective function. This finding obviously indicates that an appropriate temperature profile can be obtained and the profile adheres to the magnitude of the blood perfusion
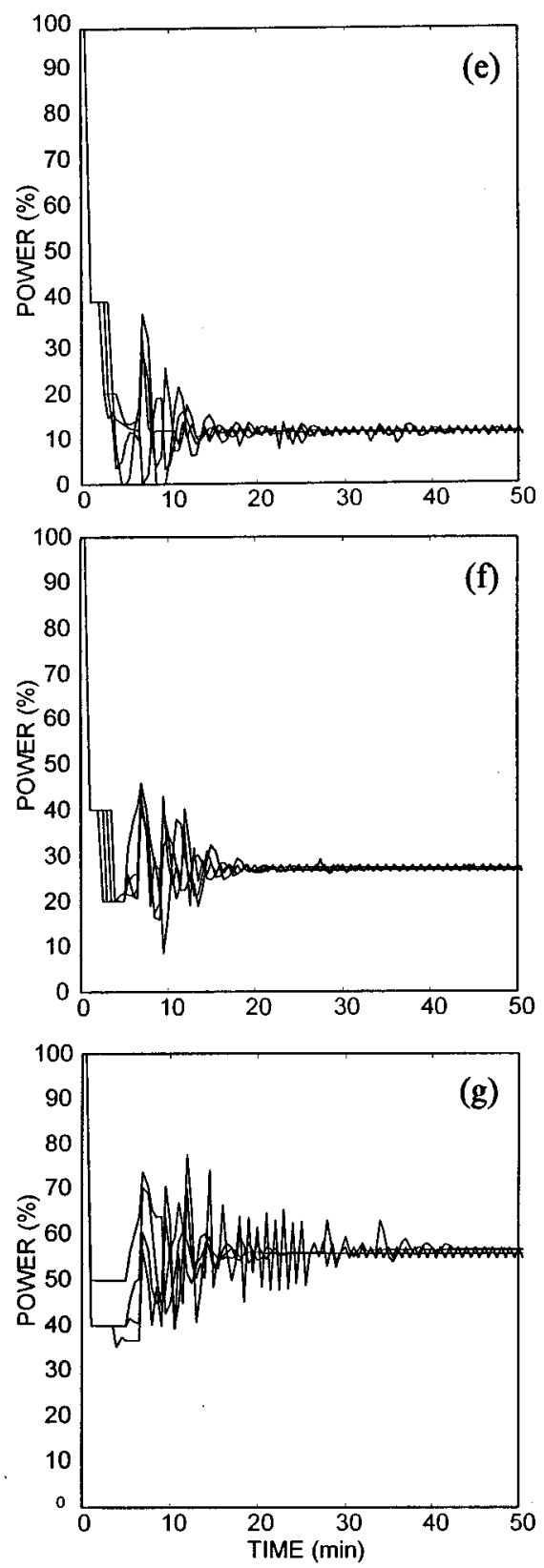

FIG. 9. Effect of the blood perfusion and the $T_{c}$ location on the objective function and the output power level. (a)-(d) are the responses of the objective functions for different blood perfusions, while $T_{c}$ is located at thermosensors 1,2,3, and 4, respectively; the three curves (from the bottom to the top) in each plot are for blood perfusion equal to 2,5 , and $10 \mathrm{~kg}$ $\mathrm{m}^{-3} \mathrm{~s}^{-1}$, respectively. (e)-(g) are the responses of output power levels for different $T_{c}$ locations, while the blood perfusion is 2,5 , and $10 \mathrm{~kg} \mathrm{~m}^{-3} \mathrm{~s}^{-1}$, respectively.

but is independent of the $T_{c}$ location as long as the thermosensor of $T_{c}$ is located in the tumor region and $T_{r}$ is determined by Eq. (5). The effect of the blood perfusion on the temperature profile and the objective function can also be 
seen in Figs. 7 and 8. The temperature profile related to the heating duration of 20-30 min is relatively more dispersed due to a higher blood perfusion $\left(20 \mathrm{~kg} \mathrm{~m}^{-3} \mathrm{~s}^{-1}\right)$, however, the temperature profile still stays in the range of $43-46{ }^{\circ} \mathrm{C}$ to achieve a minimum objective function. Figures $9(\mathrm{e})-9(\mathrm{~g})$, which show the simulation results of the response of the output power levels for different blood perfusions, reveal that the output power approaches a specific value which is related to the magnitude of the blood perfusion but independent of the $T_{c}$ location ( $T_{c}$ varied from thermosensor 1-4). A higher output power is required to overcome the energy carried away by a higher blood perfusion. A higher perfusion appears to result in a greater fluctuation of output power level and the fluctuation is also related to the $T_{c}$ location. The thermosensor of $T_{c}$ located at the location with a higher power density has a greater fluctuation of heating temperature and output power level. ${ }^{5,6}$

\section{CONCLUSION}

The paper presents an objective function-based $T_{r}$ used to adjust the reference temperature for a self-tuning fuzzy logic controller. The time-varying nature of the blood perfusion response illustrates that a controller of this design has the capability to provide appropriate regulation of ultrasound power for the scanned focused ultrasound heating system. Extensive simulation of the controller has shown that it has acceptable settling time, and that it is capable of maintaining the treatment temperatures within the desired range, compensating for the temporal variation of blood perfusion. The simulation results indicate that a control system of this design has improved performance relative to existing controllers in its robust performance, simple architecture, and algorithm, with no a priori requirement for blood perfusion identification. The simulation results using this control algorithm with $T_{r}$ based on objective functions also demonstrate that there are no significant differences for the temperature profiles for different control thermosensor $\left(T_{c}\right)$ locations and the initial setting value of the reference temperature $\left(T_{r}\right)$. In the future, the control strategy should be tested in experimental evaluations to establish its robustness for patient safety and efficacy.

\section{ACKNOWLEDGMENTS}

The authors would like to thank the National Science Council and the Department of Health of the Republic of China for partially supporting this research under Contract Nos. NSC 87-2213-E-002-075 and DOH 87-HR-635, respectively.

\footnotetext{
a) Address for correspondence: Win-Li Lin, Institute of Biomedical Engineering, College of Medicine, National Taiwan University, No. 1, Sec. 1, Jen-Ai Road, Taipei, Taiwan.
}

${ }^{1}$ R. B. Roemer, "Thermal dosimetry," in Thermal Dosimetry and Treatment Planning, edited by M. Gautherie (Springer, Berlin, 1990), pp. 119214.

${ }^{2}$ A. J. Fenn and A. K. Gerald, "Experimental investigation of an adaptive feedback algorithm for hot spot reduction in radio-frequency phased-array hyperthermia," IEEE Trans. Biomed. Eng. BME-43, 273-280 (1996).

${ }^{3}$ P. VanBaren, E. Beck, E. S. Ebbini, and C. A. Cain, "Feedback control of temperature during hyperthermia treatments with phased-array ultrasound applicators," Proceedings of the 17th Annual Conference of IEEE Eng. in Med. and Biol. and 21st Canadian Med. and Biol. Eng. Conference, Montreal, Canada, pp. 613-614 (1995).

${ }^{4}$ J. K. Potocki and H. S. Tharp, "Control strategy for hyperthermia," Proceedings of the 2nd IEEE Conference on Control Application, Vancouver, Canada, Vol. 1, pp. 427-431 (1993).

${ }^{5}$ W.-L. Lin, R. B. Roemer, and K. Hynynen, “Theoretical and experimental evaluation of a temperature controller for scanned focused ultrasound hyperthermia,', Med. Phys. 17, 615-625 (1990).

${ }^{6}$ W.-L. Lin, M. J. Shieh, R. B. Roemer, and C. Y. Wang, "Temperature control of ultrasound hyperthermia with optimal thermocouple location and target temperature,' Biomed. Eng. - Appl. Basis Comm. 5, 504-514 (1993).

${ }^{7}$ W.-L. Lin, R. B. Roemer, E. G. Moros, and K. Hynynen, “'Optimization of temperature distributions in scanned focused ultrasound hyperthermia," Int. J. Hyperthermia 8, 61-78 (1992).

${ }^{8}$ A. W. Dutton, R. B. Roemer, and F. A. Gibbs, "A clincially integrated scanned focused ultrasound hyperthermia," Proceedings of the 7th International Congress on Hyperthermic Oncology (Rome, Italy, 1996), Vol. 1, p. 92.

${ }^{9}$ E. G. Moros, Simulations of scanned focused ultrasound hyperthermia: the effects of scanning speed, scanning pattern, and multiple tilted transducers, Master thesis, University of Arizona (1987).

${ }^{10} \mathrm{H}$. H. Pennes, "Analysis of tissue and arterial blood temperatures in the resting human forearm," J. Appl. Phys. 1, 93-122 (1948).

${ }^{11}$ H. F. Bowman, "Heat transfer mechanism and thermal dosimetry," J. Natl. Cancer Inst., Monograph 61, 437-445 (1981).

${ }^{12}$ J. C. Chato, "Selected thermophysical properties of biological materials," in Heat Transfer in Medicine and Biology: Analysis and Applications, edited by A. Shitzer and R. C. Eberhart, (Plenum, New York, 1985), Vol. 2, pp. 413-418.

${ }^{13}$ R. G. Gordon, R. B. Roemer, and S. M. Horvath, "A mathematical model of the human temperature regulatory system-Transient cold exposure response,"' IEEE Trans. Biomed. Eng. BME-23, 434-444 (1976).

${ }^{14}$ R. K. Jain, "Bioheat transfer: Mathematical models of thermal systems," in Hyperthermia Cancer Therapy, edited by F. K. Storm (Hall Medical, Boston, 1983), pp. 9-46.

${ }^{15}$ J. H. Ferziger, Numerical Method for Engineering Application (Wiley, New York, 1981).

${ }^{16}$ G. D. Smith, Numerical Solution of Partial Differential Equations: Finite Difference Methods (Clarendon, Oxford, 1989).

${ }^{17}$ W. C. Daugherity, B. Rathakrishnan, and J. Yen, "Performance evaluation of a self-tuning fuzzy controller," Proceedings of the 1992 IEEE International Conference Fuzzy Systems-FUZZ-IEEE (San Diego, CA, 1992), pp. 389-397.

${ }^{18}$ C. C. Lee, "Fuzzy logic in control systems: Fuzzy logic controller-Part I,’ IEEE Trans. Syst. Man Cybern. 20, 404-418 (1990).

${ }^{19}$ D. P. Anhalt, K. Hynynen, and R. B. Roemer, "Patterns of changes of tumor temperatures during clinical hyperthermia: Implications for treatment planning, evaluation and control,"' Int. J. Hyperthermia 11, 425436 (1995).

${ }^{20}$ C. W. Song, A. Lokshina, J. G. Rhee, M. Patten, and S. H. Levitt, "'Implication of blood flow in hyperthermia treatment of tumors," IEEE Trans. Biomed. Eng. BME-31, 9-16 (1984). 